\title{
Genetic Modification Strategies to Enhance CAR T Cell Persistence for Patients With Solid Tumors
}

\section{OPEN ACCESS}

Edited by:

Avery Dexter Posey Jr.,

University of Pennsylvania,

United States

Reviewed by:

Irene Scarfo,

Massachusetts General Hospital and

Harvard Medical School,

United States

Sonia Guedan,

August Pi i Sunyer

Biomedical Research Institute

(IDIBAPS), Spain

*Correspondence:

Christopher DeRenzo

Chris.DeRenzo@stjude.org

Specialty section:

This article was submitted to

Cancer Immunity and Immunotherapy,

a section of the journal

Frontiers in Immunology

Received: 19 August 2018

Accepted: 25 January 2019

Published: 15 February 2019

Citation:

DeRenzo C and Gottschalk S (2019)

Genetic Modification Strategies to

Enhance CAR T Cell Persistence for

Patients With Solid Tumors.

Front. Immunol. 10:218.

doi: 10.3389/fimmu.2019.00218

\section{Christopher DeRenzo * and Stephen Gottschalk}

Department of Bone Marrow Transplantation and Cellular Therapy, St. Jude Children's Research Hospital, Memphis, TN, United States

Immunotherapy with chimeric antigen receptor (CAR) T cells offers a promising method to improve cure rates and decrease morbidities for patients with cancer. In this regard, CD19-specific CAR T cell therapies have achieved dramatic objective responses for a high percent of patients with CD19-positive leukemia or lymphoma. Most patients with solid tumors however, have experienced transient or no benefit from CAR T cell therapies. Novel strategies are therefore needed to improve CAR T cell function for patients with solid tumors. One obstacle for the field is limited CAR T cell persistence after infusion into patients. In this review we highlight genetic engineering strategies to improve CAR T cell persistence for enhancing antitumor activity for patients with solid tumors.

Keywords: CAR T cell, immunotherapy, persistence, genetic modification, solid tumor

\section{INTRODUCTION}

Patients with relapsed or refractory solid tumors have poor outcomes and novel treatments are needed to improve survival and decrease morbidities resulting from conventional treatments. Immunotherapy with chimeric antigen receptor (CAR) $\mathrm{T}$ cells is a promising strategy to improve these outcomes. Generally, CARs consist of three components: (i) a genetically engineered receptor capable of recognizing a tumor associated antigen (TAA) in an HLA-independent manner, (ii) an activation domain derived from the $\mathrm{CD} 3 \zeta$ chain of the $\mathrm{T}$ cell receptor complex, and (iii) costimulatory domain(s) to potentiate activation initiated via CAR-CD3 $\zeta$ signaling (Figure 1A). CARs are categorized as 1st generation if the construct has no costimulatory domain, 2nd generation when incorporating 1 costimulatory domain, and 3rd generation when incorporating two costimulatory domains (Figure 1A) (1). CAR T cells require three signals for optimal effector function: (i) activating signal induced by CAR recognition of tumor antigen and subsequent signal transduction through $\mathrm{CD} 3 \zeta$, (ii) costimulation provided via one or more domains engineered into the CAR construct, and (iii) stimulatory cytokines for continued growth and effector function (Figure 1B). 

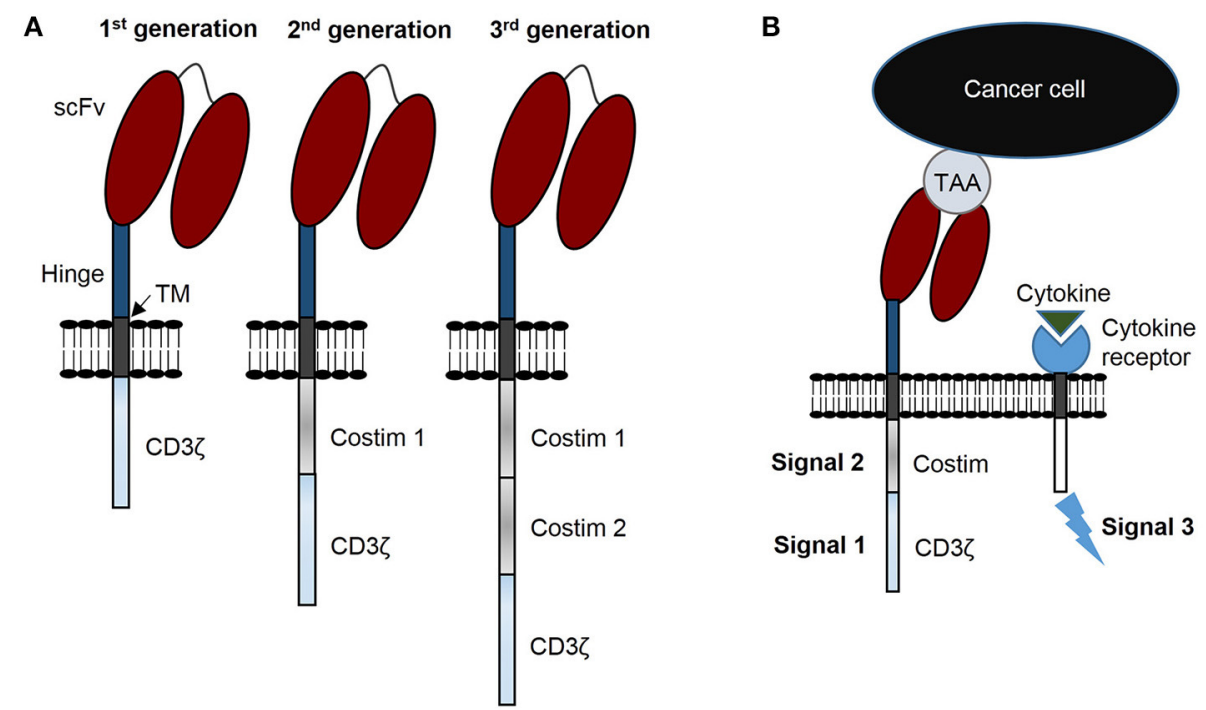

FIGURE 1 | CAR terminology: components, generations, and signals. (A) CARs consist of 3 components: a single chain variable fragment (scFv) that recognizes tumor associated antigen (TAA), costimulatory domain(s), and a CD3 $\zeta$ domain. CARs are designated 1st generation with no costimulatory domain, 2nd generation

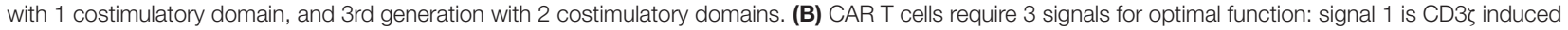
activation, signal 2 costimulation, and signal 3 functional augmentation via stimulatory cytokine(s). TM, transmembrane domain.

While CAR T cell therapy offers promise to improve outcomes for patients with difficult to treat cancers, strategies must be improved to benefit large numbers of patients suffering from relapsed and refractory solid tumors. Several obstacles need to be addressed to realize this goal, including selection of optimal TAAs (2-4), T cell homing to sites of malignant disease $(5,6)$, $\mathrm{T}$ cell penetration into solid masses $(7,8)$, and overcoming the immunosuppressive tumor microenvironment (9-11). Enhancing persistence of adoptively transferred $\mathrm{T}$ cells is another vital challenge for successful treatment of cancer (12-14). Multiple strategies exist to enhance CAR T cell persistence against solid tumors including pre-treatment with cytoreductive chemotherapy (15), optimized $\mathrm{T}$ cell culture conditions (16), $\mathrm{T}$ cell selection procedures $(17,18)$, and combinatorial treatment approaches (19-24). This review is focused on novel genetic engineering strategies to enhance CAR $T$ cell persistence and antitumor activity against solid tumors. While promising preclinical data is available, only few of these approaches have been evaluated in early phase clinical studies.

\section{NOVEL COSTIMULATION STRATEGIES TO IMPROVE PERSISTENCE AND ANTI-SOLID TUMOR ACTIVITY}

Second generation CAR constructs with CD28 or $41 \mathrm{BB}$ costimulatory endodomains are most frequently utilized to generate CAR T cells. Comparison of CD28 vs. $41 \mathrm{BB}$ to enhance the function of CAR $\mathrm{T}$ cells has been reviewed previously (25). Investigators are now actively exploring CARs encoding alternative costimulatory endodomains or strategies to provide costimulation with a 2nd molecule expressed in CAR T cells.
Recent findings demonstrate that CAR $\mathrm{T}$ cell persistence can be enhanced against solid tumors by transducing CD4 and CD8 T cells with CARs encoding different costimulatory domains (Figure 2A) (26). This was demonstrated by separately transducing CD4 and CD8 $\mathrm{T}$ cells with mesothelin-specific CARs containing either a CD28, 41BB, or ICOS costimulatory domain. After separate transductions, CD4- and CD8-CAR T cells were mixed and infused to determine the optimal combination for enhanced persistence against lung cancer in vivo. Strikingly, the best combination for enhanced persistence of CD8-CAR $\mathrm{T}$ cells resulted from mixing CD4.ICOS- and CD8.41BB-CAR $\mathrm{T}$ cells before injection. CD4.ICOS-CAR $\mathrm{T}$ cells persisted in vivo regardless of the costimulatory domain expressed by CD8CAR T cells. On the other hand, CD4-CAR T cells expressing either a CD28 or $41 \mathrm{BB}$ costimulatory domain had minimal persistence under any condition, clearly demonstrating that the costimulatory domain of CD4-CAR T cells affects persistence of both CD4- and CD8-CAR T cells in this model. Based on these data, the authors generated a 3rd generation ICOS.41BBCAR, which also led to enhanced persistence of both CD4and CD8-CAR $\mathrm{T}$ cells in vivo, and greater antitumor activity compared to 2nd generation CAR T cells. Given that CD4 T cells provide signals to enhance persistence and effector function of cytotoxic CD8 T cells, this concept is highly relevant for development of future CAR T cell trials for patients with solid tumors. Furthermore, evidence demonstrates that, at least in some models, CD4-CAR T cells targeting solid tumors persist longer in vivo and result in superior antitumor activity compared to CD8-CAR T cells (27). Given that optimal costimulatory domains may be different for CD4- and CD8-CAR T cells, CD4CAR T cells can directly kill cancer cells, and single vectors can be used to make 3 rd generation CARs for enhancing persistence 
A

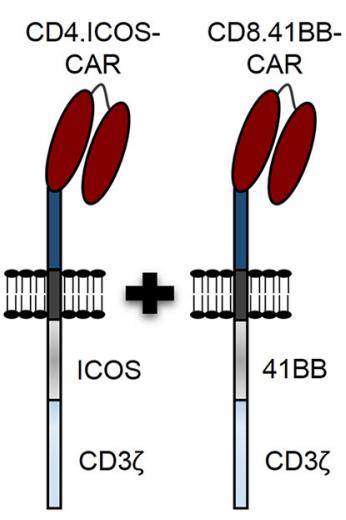

D

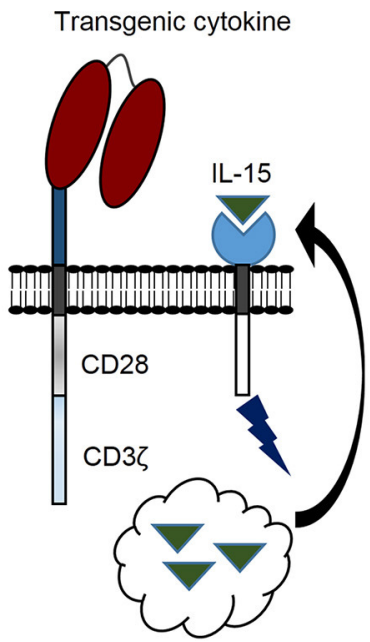

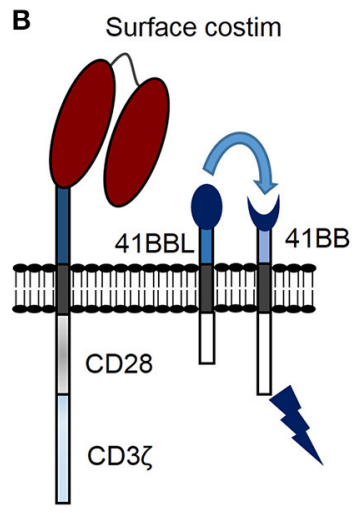

E
C

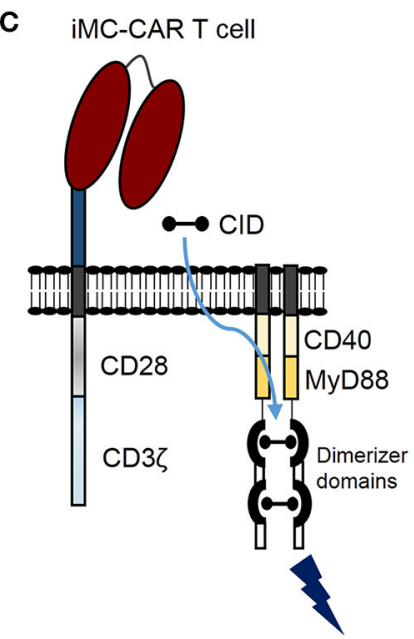

Transgenic cytokine receptor
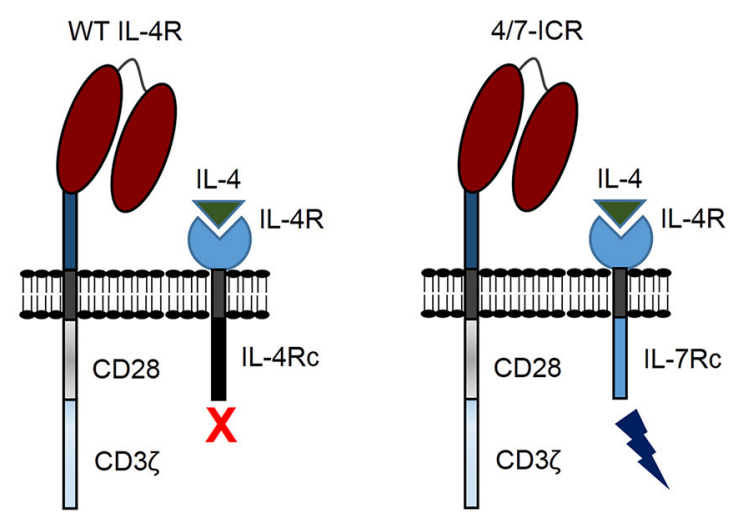

FIGURE 2 | Novel genetic modifications to enhance CAR T cell persistence against solid tumors. (A) CAR T cell persistence against solid tumors can be enhanced by transducing CD4 and CD8T cells with different CAR costimulatory domains. (B) CAR T cell activation leads to 41BB receptor (41BB) expression. CAR T cell persistence can be enhanced by constitutive expression of 41BB ligand (41BBL), which interacts with the 41BB receptor in an autocrine or paracrine manner to provide additional costimulatory signal (surface costim). (C) Triggering CAR T cell costimulation with a chemical inducer of dimerization (CID) drug is another promising approach. A molecule, which consists of two dimerizer domains and costimulatory domains derived from MyD88 and CD40 (iMC) can be activated by CID and enhance CAR T cell persistence. (D) Constitutively expressed cytokine (i.e., IL-15) can be engineered by tethering cytokine to the cell membrane (not shown) or secreted by CAR T cells (black arrow) to enhance persistence. (E) The inverted IL-4/IL-7 chimeric cytokine receptor (4/7-ICR) contains an IL-4 receptor extracellular domain (IL-4R) fused to an activating IL-7 cytoplasmic domain (IL-7Rc). While IL-4 binding to wild type (WT) IL-4 receptor leads to CAR T cell inhibition, IL-4 binding to 4/7-ICR enhances persistence and effector function.

of both CD4- and CD8-CAR T cells, future studies focused on optimizing costimulation for both CD4- and CD8-CAR T cells should benefit patients with solid tumors.

Providing costimulation through a 2nd molecule is another promising strategy to enhance persistence, antitumor activity, and safety of CAR $\mathrm{T}$ cells for patients with solid tumors. For example, activated $\mathrm{T}$ cells express the $41 \mathrm{BB}$ receptor, and investigators have shown that CD28-CAR $\mathrm{T}$ cells expressing 41BB ligand on the cell surface (Figure 2B) endows $\mathrm{T}$ cells with superior effector function in comparison to $\mathrm{T}$ cells expressing a traditional 3rd generation CD28.41BB-CAR (28). Expressing other tumor necrosis factor superfamily ligands on the T cell surface, such as CD40 ligand, has also resulted in improved CAR $\mathrm{T}$ cell function (29).
Triggering CAR T cell costimulation with a drug is another promising approach. For example, investigators designed a molecule, which consists of two dimerizer domains and costimulatory domains derived from MyD88 and CD40 (iMC) (Figure 2C) and can be activated by a chemical inducer of dimerization (CID) (30). While initially, this molecule was used to activate dendritic cells, two subsequent studies, as delineated in the next section, have highlighted the benefit of iMC in CAR T cells $(31,32)$. Of note, costimulation is only activated in the presence of CID, providing a safety mechanism to limit $\mathrm{T}$ cell activation.

Using 1st generation prostate-specific antigen (PSCA)-CAR T cells containing an iMC domain (PSCA-iMC-CAR) and PSCApositive pancreatic cancer cells in vitro, Foster and colleagues 
demonstrated that CID induced costimulation resulted in greater CAR T cell cytokine secretion, proliferation, and antitumor activity compared to the same cells without CID. In vivo, PSCA.iMC-CAR $\mathrm{T}$ cells were used to treat mice harboring pancreatic cancers plus/minus weekly CID. Indeed, CID administration enhanced PSCA.iMC-CAR antitumor activity, and 21 days post $\mathrm{T}$ cell injection CAR $\mathrm{T}$ cells were detected in the blood and tumors from mice treated with CID, but not in those treated without CID.

Separately, Mata and colleagues generated HER2-specific CAR T cells incorporating an inducible costimulatory domain (HER2.iMC-CAR) containing both MyD88 and CD40 signaling regions, (32) and demonstrated that HER2.iMC-CAR T cells have enhanced effector function compared to 2 nd generation HER2.CD28-CAR $\mathrm{T}$ cells utilized in clinical trials (33). Importantly, in repeat stimulation assays, which model CAR T cell persistence against solid tumors in vitro, HER2.iMC-CAR $\mathrm{T}$ cells expanded 100-1,000 fold greater than 2 nd generation HER2-CAR T cells when targeting multiple different HER2positive solid tumor types. In vivo, HER2.iMC-CAR T cells had significantly greater antitumor activity that led to an enhanced survival benefit compared to 2nd generation HER2-CAR T cells, and controlled solid tumors at a very low $\mathrm{T}$ cell dose. Furthermore, repeated induction of costimulation via multiple doses of CID led to superior antitumor activity and a survival advantage for mice bearing HER2-positive solid tumors.

In summary, these results demonstrate that non-traditional costimulatory domains can enhance CAR T cell persistence and antitumor activity against solid tumors, and an inert drug can be injected to adjust CAR $\mathrm{T}$ cell activation over time.

\section{TRANSGENIC CYTOKINES ENHANCE CAR T CELL EFFECTOR FUNCTION AGAINST SOLID TUMORS}

In addition to modifying costimulatory domains, genetic modification can be used to endow CAR T cells with the ability to express stimulatory cytokines. Interleukin (IL)-15, IL-12, and IL-18 are three examples under active exploration. Constitutively expressed IL-15 can be engineered by tethering IL-15 to the cell membrane (34) or secreted by modified T cells (Figure 2D) (35). In this regard, IL-13R $\alpha 2$-CAR T cells modified to secrete IL15 (IL-13R $\alpha 2 . I L-15-C A R$ ) demonstrated superior proliferative capacity and antitumor activity in vitro and in vivo against high grade glioma compared to IL-13R $\alpha 2$-CAR alone (35). Both IL13R $\alpha 2$ - and IL-13R $\alpha 2$.IL-15-CAR T cells had comparable in vivo antitumor activity up to 4 weeks; however, after 4 weeks IL-15 expressing CAR $\mathrm{T}$ cells had greater activity indicating that IL15 improved $\mathrm{T}$ cell persistence over a prolonged period of time. Indeed, IL-15 expressing CAR T cells were detected in vivo for a significantly longer period of time compared to CAR alone. Intriguingly, in mice treated with IL13-R $\alpha 2$.IL-15-CAR T cells, tumors recurred at late time points and the majority of relapsed tumors no longer expressed IL-13R $\alpha 2$, implicating antigen loss as a tumor escape mechanism in this model. This predicts that despite the benefits of improving CAR T cell persistence against solid tumors, antigen loss variants can occur, and strategies to target solid tumors in future clinical trials may require targeting multiple tumor antigens $(36,37)$. Clinically, transgenic IL-15 expression is actively being explored to improve expansion, persistence and antitumor activity of GD2-CAR invariant natural killer cells for the treatment of patients with neuroblastoma (NCT03294954). Results from this trial should provide insight regarding the impact of constitutively secreted IL-15 to enhance persistence and function of adoptively transferred CAR modified cells, and determine safety in the clinical setting.

IL-12 is another promising cytokine under active exploration to enhance CAR T cell persistence and effector function in both preclinical models (38-40) and a phase I clinical trial for patients with solid tumors (NCT02498912). To enhance CAR $\mathrm{T}$ cell activity against ovarian cancer, 2nd generation MUC16 ${ }^{\text {ecto }}$-specific CAR T cells were modified to secrete IL-12 (MUC16 $6^{\text {ecto }}$.IL-12-CAR) (40). In vivo MUC16 ${ }^{\text {ecto }}$.IL12-CAR T cells demonstrated superior antitumor activity and were detected in the peripheral blood of treated animals, while the same CAR T cells without IL-12 were not detected at any time point, indicating that constitutive IL-12 secretion increased CAR T cell persistence against ovarian cancer. A clinical trial is underway investigating MUC16 ${ }^{\text {ecto }}$.IL-12-CAR T cells for patients with MUC16 ${ }^{\text {ecto }}$ positive tumors (NCT02498912), and results should shed light on the possibility of translating this technique to treat a broad range of patients afflicted with solid tumors.

CAR T cells genetically modified to secrete IL-18 exhibit superior antitumor activity against solid tumors compared to 2 nd generation CAR T cells in pre-clinical models. Chmielewski and Abken compared 2nd generation CEA-CAR T cells containing a CD28 costimulatory domain to CEA-CAR T cells modified to secrete IL-18 (CEA.IL-18-CAR) under control of a nuclear factor of activated T cells (NFAT)-IL-2 minimal promoter (41). Placing cytokine secretion under control of the NFAT-IL-2 promoter creates an inducible system, whereas cytokine is only secreted upon $\mathrm{T}$ cell recognition of its target antigen, theoretically limiting cytokine secretion to the tumor environment. In an immunecompetent model of bulky CEA-positive pancreatic cancer, a single injection of CEA.IL-18-CAR $\mathrm{T}$ cells led to prolonged survival compared to mice treated with 2 nd generation CEACAR. Prolonged survival and enhanced antitumor activity were attributed to a pro-inflammatory environment induced by CAR mediated IL-18 secretion. Compared to tumors treated with 2nd generation CEA-CAR, tumors obtained after CEA.IL-18CAR treatment demonstrated an increased quantity of proinflammatory natural killer cells and M1 macrophages, and a decreased quantity of anti-inflammatory M2 macrophages, regulatory $\mathrm{T}$ cells, and CD103-positive dendritic cells. Other groups have shown enhanced antitumor activity by genetically modifying T cells to secrete IL-18 $(42,43)$, and this strategy merits further exploration to enhance CAR T cell activity against solid tumors.

Stimulatory cytokine pathways can also be constitutively activated without the need for cytokine induced stimulation, thus providing $\mathrm{T}$ cell survival signals when no cytokine is in the milieu. To enhance expansion, persistence and antitumor activity of 2nd generation GD2-CAR $\mathrm{T}$ cells against neuroblastoma, 
investigators modified CAR T cells with a constitutively active IL-7 cytokine receptor (C7R) that lacks the IL-7 receptor extracellular domain (44). C7R modified CAR T cells were able to proliferate and kill neuroblastoma cells in serial killing assays to a greater degree than GD2-CAR T cells alone. Impressively, at a low $\mathrm{T}$ cell dose GD2.C7R-CAR $\mathrm{T}$ cells had substantial antitumor activity in vivo against metastatic neuroblastoma. Comparatively, GD2-CAR T cells had limited antitumor activity at the same low $\mathrm{T}$ cell dose. Improved antitumor activity resulted in enhanced survival that was secondary to increased expansion and persistence of C7R expressing CAR T cells. Mechanistically, GD2-CAR T cells expressing C7R had greater cell division and reduced apoptosis compared to GD2-CAR alone, effects attributed, in part, to increased BCL2 expression.

While CAR $\mathrm{T}$ cell transgenic cytokine production and signaling offer a promising strategy to improve persistence and function of CAR T cells for patients with solid tumors, safety concerns exist. T cell autonomous growth (45) and cytokine induced toxicity can occur (46), and these concerns need to be considered for treating patients. Notably, for both IL13R $\alpha 2$.IL15-CAR and MUC16 ${ }^{\text {ecto }}$.IL-12-CAR T cells, IL-15 or IL-12 production was low at baseline and increased multiple fold in an antigen specific manner, indicating that elevated cytokine levels should be limited to the local tumor environment. Additionally, both aforementioned CARs were modified with a "safety switch" that proved capable of efficiently eliminating gene modified cells. IL-13R $\alpha 2$.IL-15-CAR T cells were modified with an inducible caspase 9 (iC9) safety switch that initiates apoptosis in the presence of CID, and these T cells were efficiently eliminated by CID induced iC9 activation. Importantly, the iC9 safety switch was demonstrated to be effective in the clinical setting (47) making it a viable safety mechanism for future trials. MUC16 ${ }^{\text {ecto }}$.IL-12-CAR T cells were modified with a truncated epidermal growth factor receptor that can be targeted by cetuximab, a clinically available monoclonal antibody.

As discussed previously, IL-18 secretion in CEA-CAR T cells was under control of an NFAT-IL-2 minimal promoter, which enables T cells to secrete IL-18 only in the presence of target antigen. For CEA.IL-18-CAR T cells, IL-18 was secreted at high levels in the presence of CEA-positive pancreatic cancer cells, but only minimal IL-18 was detected when T cells were exposed to CEA-negative pancreatic cancer cells. Although this strategy demonstrated benefit in this and other pre-clinical studies (48), initial experience in humans was less promising. In one clinical study, the adoptive transfer of ex vivo expanded tumor infiltrating lymphocytes transduced to secrete IL-12 under the control of an NFAT-IL-2 minimal promoter induced significant toxicities attributed to high serum IL-12 levels in patients with melanoma (49).

In regard to safety for $\mathrm{C} 7 \mathrm{R}$ modified CAR $\mathrm{T}$ cells, these demonstrated no cell autonomous growth, and included an iC9 safety switch that led to efficient GD2.C7R-CAR T cell elimination when treated with CID. Alternate gene modification strategies can also be utilized to enhance the safety of CAR $\mathrm{T}$ cells by enabling full CAR $\mathrm{T}$ cell activation only in the presence of 2 TAAs. Examples include chimeric costimulatory receptors $(50,51)$ and synthetic Notch receptors (52). Another novel method is to simultaneously induce CAR T cell STAT5 signaling, analogous to signal provided by IL-2, IL-7, or IL-15, and STAT3 signaling, analogous to signal provided by IL-21, in an antigen dependent manner. In this regard, Kagoya and colleagues developed a "new generation" CD19-CAR containing a CD28 costimulatory domain, a truncated IL-2R $\beta$ domain to activate STAT5, and a C-terminus YXXQ motif to activate STAT3 (53). Importantly, STAT5 and STAT3 activation occurred in an antigen dependent manner and the new generation CAR enhanced proliferation of both CD4- and CD8-CAR T cells in vitro and in vivo compared to 2 nd generation CAR $\mathrm{T}$ cells with either CD28 or 41BB costimulatory domains. In an in vivo solid tumor model using A375 melanoma cells modified to express CD19, new generation CAR T cells persisted to a greater degree in tumors and peripheral blood of treated animals, and had greater antitumor activity compared to 2 nd generation CAR T cells.

Together, these findings demonstrate that while safety concerns exist, strategies can be implemented to eliminate genetically modified cells in the setting of unacceptable toxicity, limit full CAR $\mathrm{T}$ cell activation to sites of tumor expressing two or more TAAs, or deliver cytokine signals to CAR T cells via activation of STAT5 and STAT3 in an antigen dependent manner.

In summary, studies demonstrate that CAR $\mathrm{T}$ cells can be safely engineered to express transgenic cytokines or constitutively active cytokine receptors, which impart CAR $\mathrm{T}$ cells with enhanced persistence and antitumor activity against solid tumors. Active and future clinical trials implementing these techniques will guide strategies to improve outcomes for patients with solid tumors.

\section{TRANSGENIC CYTOKINE RECEPTORS TO OVERCOME TUMOR SECRETED INHIBITORY MILIEU}

Overcoming effects of inhibitory cytokines is another strategy to enhance CAR T cell persistence and function for treating patients with solid tumors. Switch receptors are one way to transform inhibitory cytokine signals into a stimulus, and thus increase $\mathrm{T}$ cell persistence and function (Figure 2E) $(54,55)$. Effectiveness of this strategy was demonstrated against pancreatic cancer using a PSCA-specific CAR engineered with an inverted IL-4/IL-7 chimeric cytokine receptor (4/7-ICR) (56), which contains an IL-4 extracellular domain fused to an activating IL-7 intracellular domain. PSCA-CAR T cells initially killed PSCApositive pancreatic cancer cells, but only PSCA.4/7-ICR-CAR $\mathrm{T}$ cells killed and expanded in the presence of both pancreatic cancer cells and the inhibitory cytokine IL-4. This held true in vivo where PSCA.4/7-ICR-CAR T cells exhibited enhanced antitumor activity and greater expansion compared to CAR T cells without 4/7-ICR. Importantly, once PSCA-positive solid tumor cells were eliminated, PSCA.4/7-ICR-CAR T cells no longer expanded, indicating no cell autonomous growth and a positive safety profile. This strategy was also utilized to target breast cancer using 2nd generation MUC1-CAR T cells (57). MUC1.4/7-ICR-CAR T cells demonstrated enhanced expansion 
and antitumor activity in vitro and in vivo compared to MUC1CAR alone, demonstrating that the 4/7-ICR enhances CAR T cell persistence and function against multiple solid tumor types in the presence of the inhibitory cytokine IL-4.

Transgenic cytokine receptors can also be combined to impart CAR $\mathrm{T}$ cells with the ability to transform multiple inhibitory signals into different types of T cell stimuli. Transgenic cytokine receptors were previously developed to overcome the inhibitory effect of transforming growth factor $\beta$ (TGF $\beta$ ), a potent immunosuppressive cytokine secreted by multiple solid tumor types. Dominant negative TGF $\beta$ receptors (DNR) enable $\mathrm{T}$ cells to avoid effects exerted by $\operatorname{TGF} \beta(58,59)$, and DNR transduced tumor-specific $T$ cells were recently shown to safely persist in patients years after $\mathrm{T}$ cell infusion (60). TGF $\beta$ receptor II extracellular domains can also be fused with stimulatory intracellular domains such as the 41BB endodomain (TGF $\beta / 41 \mathrm{BB})$. In a recent publication 1st generation PSCA-CAR $\mathrm{T}$ cells were modified to express both TGF $\beta / 41 \mathrm{BB}$ and $4 / 7$ ICR, and dubbed SmarT cells (61). SmarT cells demonstrated the ability to recognize PSCA antigen on pancreatic cancer cells through the CAR, induce costimulation via TGF $\beta$ induced activation of $41 \mathrm{BB}$, and initiate cytokine signaling through 4/7-ICR in the presence of IL-4. Triple genetic modification enabled SmarT cells to specifically recognize PSCA-positive pancreatic cancer cells, expand, persist, and kill tumor cells in an immunosuppressive TGF $\beta$ and IL-4 rich environment. Importantly continued SmarT cell expansion and antitumor activity were dependent upon both PSCA antigen positivity and cytokine induced stimulation through the transgenic receptors. In vivo, SmarT cells expanded, persisted, and eliminated pancreatic tumors expressing PSCA, TGF $\beta$, and IL-4, with minimal expansion/persistence at tumor sites expressing PSCA only. Once PSCA-positive, TGF $\beta$, and IL-4 secreting solid tumors were eliminated, SmarT cells contracted, further demonstrating safety of this approach.

In conjunction with enhancing CAR $\mathrm{T}$ cell persistence and effector function, transgenic cytokine receptors can be used for selectively enhancing growth of transduced CAR T cells during the $\mathrm{T}$ cell manufacturing process. In this regard, a $4 \alpha \beta$ chimeric cytokine receptor was developed, consisting of an extracellular IL- $4 \mathrm{R} \alpha$ domain paired with an endodomain derived from the shared IL-2/IL-15 $\beta$ chain (62). This strategy was demonstrated to be effective for generating CAR T cells to target multiple solid tumor types in pre-clinical studies $(20,63)$, and is under active investigation in a clinical trial for patients with head and neck squamous cell carcinoma (64) (NCT01818323).

In summary, transgenic cytokine receptors can be utilized to avoid immunosuppressive effects of inhibitory cytokines, transform inhibitory signals into activating signals, and to selectively grow transduced solid tumor specific CAR T cells.

\section{CHALLENGES AHEAD}

We have described novel genetic modification strategies to enhance the expansion and persistence of CAR $\mathrm{T}$ cells for patients with solid tumors. The next frontier in this regard is to overcome limitations imposed by current gene modification techniques, and endow CAR T cells with even greater functional capacity. CAR $\mathrm{T}$ cells face many obstacles including homing to and penetrating into solid masses, overcoming tumor antigen escape, surviving within the hostile tumor environment of low $\mathrm{pH}$, high lactate and adenosine, hypoxia, and numerous other immunosuppressive factors including inhibitory stromal cells. Realistically, current engineering strategies allow up to 3 genetic modifications to enhance CAR $\mathrm{T}$ cell function. While individual genetic modifications demonstrate efficacy to combat many of the listed challenges, the field is tasked with developing new strategies to combine a greater number of mechanisms for enabling individual CAR $\mathrm{T}$ cell products to overcome the potent solid tumor inhibitory environment. One intriguing strategy is to physically load CAR $\mathrm{T}$ cells with nanogel "backpacks" capable of delivering relatively large quantities of protein upon CAR recognition of target antigen (65). In such a paradigm, CAR T cells could be physically loaded with proliferative cytokines, leaving room to utilize genetic modifications to overcome other obstacles, such as targeting multiple solid tumor antigens. Another promising strategy is to utilize targeted gene editing and insert CAR constructs into $\mathrm{T}$ cell inhibitory loci $(66,67)$. For instance, CAR DNA could be inserted into the adenosine 2 a receptor locus (68), decreasing CAR T cell immunosuppression in an adenosine rich milieu. As these, and other novel strategies are implemented, we envision CAR $\mathrm{T}$ cells capable of safely persisting long term and overcoming a greater number of tumor immune evasion strategies.

\section{CONCLUSIONS}

Several strategies have been developed to enhance CAR T cell expansion, persistence and antitumor activity by introducing novel costimulatory domains, cytokine genes, and constitutively active or inverted cytokine receptors into $\mathrm{T}$ cells. While there are safety concerns regarding autonomous cell growth and cytokine induced toxicity using these approaches, encouraging efficacy and safety data from preclinical studies supports continued preclinical testing and evaluation in humans. Thus, we remain hopeful that optimized CAR $\mathrm{T}$ cells will eventually improve outcomes and decrease toxicities for patients suffering from solid tumors.

\section{AUTHOR CONTRIBUTIONS}

All authors listed have made a substantial, direct and intellectual contribution to the work, and approved it for publication.

\section{ACKNOWLEDGMENTS}

The authors were supported by NIH grants 5T32HL092332 and 1R01CA173750, and CRPIT grant RP101335. 


\section{REFERENCES}

1. Fesnak AD, June $\mathrm{CH}$, Levine BL. Engineered T cells: the promise and challenges of cancer immunotherapy. Nat Rev Cancer (2016) 16:566-81. doi: $10.1038 /$ nrc. 2016.97

2. Orentas RJ, Yang JJ, Wen X, Wei JS, Mackall CL, Khan J. Identification of cell surface proteins as potential immunotherapy targets in 12 pediatric cancers. Front Oncol. (2012) 2:194. doi: 10.3389/fonc.2012.00194

3. Johnson LA, Scholler J, Ohkuri T, Kosaka A, Patel PR, Mcgettigan SE, et al. Rational development and characterization of humanized anti-EGFR variant III chimeric antigen receptor T cells for glioblastoma. Sci Transl Med. (2015) 7:275ra222. doi: 10.1126/scitranslmed.aaa4963

4. Posey AD Jr, Schwab RD, Boesteanu AC, Steentoft C, Mandel U, Engels B, et al. Engineered CAR T cells targeting the cancer-associated Tn-Glycoform of the membrane Mucin MUC1 control adenocarcinoma. Immunity (2016) 44:1444-54. doi: 10.1016/j.immuni.2016.05.014

5. Kershaw MH, Wang G, Westwood JA, Pachynski RK, Tiffany HL, Marincola FM, et al. Redirecting migration of T cells to chemokine secreted from tumors by genetic modification with CXCR2. HumGene Ther. (2002) 13:1971-80. doi: 10.1089/10430340260355374

6. Craddock JA, Lu A, Bear A, Pule M, Brenner MK, Rooney CM, et al. Enhanced tumor trafficking of GD2 chimeric antigen receptor T cells by expression of the chemokine receptor CCR2b. J Immunother. (2010) 33:780-8. doi: 10.1097/CJI.0b013e3181ee6675

7. Caruana I, Savoldo B, Hoyos V, Weber G, Liu H, Kim ES, et al. Heparanase promotes tumor infiltration and antitumor activity of CAR-redirected $\mathrm{T}$ lymphocytes. Nat Med. (2015) 21:524-9. doi: 10.1038/nm.3833

8. Bocca P, Di Carlo E, Caruana I, Emionite L, Cilli M, De Angelis B, et al. Bevacizumab-mediated tumor vasculature remodelling improves tumor infiltration and antitumor efficacy of GD2-CAR $\mathrm{T}$ cells in a human neuroblastoma preclinical model. OncoImmunology (2018) 7:e1378843. doi: 10.1080/2162402X.2017.1378843

9. Gajewski TF, Meng Y, Blank C, Brown I, Kacha A, Kline J, et al. Immune resistance orchestrated by the tumor microenvironment. Immunol Rev. (2006) 213:131-45. doi: 10.1111/j.1600-065X.2006.00442.x

10. Rabinovich GA, Gabrilovich D, Sotomayor EM. Immunosuppressive strategies that are mediated by tumor cells. Annu Rev Immunol. (2007) 25:267-96. doi: 10.1146/annurev.immunol.25.022106.141609

11. O'rourke DM, Nasrallah MP, Desai A, Melenhorst JJ, Mansfield K, Morrissette JJD, et al. A single dose of peripherally infused EGFRvIIIdirected CAR T cells mediates antigen loss and induces adaptive resistance in patients with recurrent glioblastoma. Sci Transl Med. (2017) 9:eaaa0984. doi: 10.1126/scitranslmed.aaa0984

12. Robbins PF, Dudley ME, Wunderlich J, El-Gamil M, Li YF, Zhou J, et al. Cutting edge: persistence of transferred lymphocyte clonotypes correlates with cancer regression in patients receiving cell transfer therapy. J Immunol. (2004) 173:7125-30. doi: 10.4049/jimmunol.173.12.7125

13. Louis CU, Savoldo B, Dotti G, Pule M, Yvon E, Myers GD, et al. Antitumor activity and long-term fate of chimeric antigen receptorpositive $\mathrm{T}$ cells in patients with neuroblastoma. Blood (2011) 118:6050-6. doi: 10.1182/blood-2011-05-354449

14. Maude SL, Frey N, Shaw PA, Aplenc R, Barrett DM, Bunin NJ, et al. Chimeric antigen receptor $\mathrm{T}$ cells for sustained remissions in leukemia. $N$ Engl J Med. (2014) 371:1507-17. doi: 10.1056/NEJMoa1407222

15. Gattinoni L, Finkelstein SE, Klebanoff CA, Antony PA, Palmer DC, Spiess PJ, et al. Removal of homeostatic cytokine sinks by lymphodepletion enhances the efficacy of adoptively transferred tumor-specific CD8+ T cells. J Exp Med. (2005) 202:907-12. doi: 10.1084/jem.20050732

16. Xu Y, Zhang M, Ramos CA, Durett A, Liu E, Dakhova O, et al. Closely related T-memory stem cells correlate with in vivo expansion of CAR.CD19$\mathrm{T}$ cells and are preserved by IL-7 and IL-15. Blood (2014) 123:3750-9. doi: 10.1182/blood-2014-01-552174

17. Klebanoff CA, Gattinoni L, Restifo NP. Sorting through subsets: which T-cell populations mediate highly effective adoptive immunotherapy? J Immunother. (2012) 35:651-60. doi: 10.1097/CJI.0b013e31827806e6

18. Themeli M, Rivière I, Sadelain $M$. New cell sources for $T$ cell engineering and adoptive immunotherapy. Cell Stem Cell (2015) 16:357-66. doi: 10.1016/j.stem.2015.03.011
19. John LB, Devaud C, Duong CP, Yong CS, Beavis PA, Haynes NM, et al. Anti-PD-1 antibody therapy potently enhances the eradication of established tumors by gene-modified T cells. Clin Cancer Res. (2013) 19:5636-46. doi: 10.1158/1078-0432.CCR-13-0458

20. Parente-Pereira AC, Whilding LM, Brewig N, Van Der Stegen SJ, Davies DM, Wilkie S, et al. Synergistic chemoimmunotherapy of epithelial ovarian cancer using ErbB-retargeted T cells combined with carboplatin. J Immunol. (2013) 191:2437-45. doi: 10.4049/jimmunol.1301119

21. Nishio N, Diaconu I, Liu H, Cerullo V, Caruana I, Hoyos V, et al. Armed oncolytic virus enhances immune functions of chimeric antigen receptor-modified T cells in solid tumors. Cancer Res. (2014) 74:5195-205. doi: 10.1158/0008-5472.CAN-14-0697

22. Khalil DN, Smith EL, Brentjens RJ, Wolchok JD. The future of cancer treatment: immunomodulation, CARs and combination immunotherapy. Nat Rev Clin Oncol. (2016) 13:273-90. doi: 10.1038/nrclinonc.2 016.25

23. Guedan S, Alemany R. CAR-T cells and oncolytic viruses: joining forces to overcome the solid tumor challenge. Front Immunol. (2018) 9:2460. doi: 10.3389/fimmu.2018.02460

24. Watanabe K, Luo Y, Da T, Guedan S, Ruella M, Scholler J, et al. Pancreatic cancer therapy with combined mesothelin-redirected chimeric antigen receptor T cells and cytokine-armed oncolytic adenoviruses. JCI Insight (2018) 3:99573. doi: 10.1172/jci.insight.99573

25. Van Der Stegen SJC, Hamieh M, Sadelain M. The pharmacology of secondgeneration chimeric antigen receptors. Nat Rev Drug Discov. (2015) 14:499509. doi: $10.1038 / \mathrm{nrd} 4597$

26. Guedan S, Posey AD Jr, Shaw C, Wing A, Da T, Patel PR, et al. (2018). Enhancing CAR T cell persistence through ICOS and 4-1BB costimulation. JCI Insight 3:96976. doi: 10.1172/jci.insight.96976

27. Wang D, Aguilar B, Starr R, Alizadeh D, Brito A, Sarkissian A, et al. Glioblastoma-targeted CD4+ CAR T cells mediate superior antitumor activity. JCI Insight (2018) 3:99048. doi: 10.1172/jci.insight.99048

28. Zhao Z, Condomines M, Van Der Stegen SJC, Perna F, Kloss CC, Gunset $\mathrm{G}$, et al. Structural design of engineered costimulation determines tumor rejection kinetics and persistence of CAR T cells. Cancer Cell (2015) 28:41528. doi: 10.1016/j.ccell.2015.09.004

29. Curran KJ, Seinstra BA, Nikhamin Y, Yeh R, Usachenko Y, Van Leeuwen DG, et al. Enhancing antitumor efficacy of chimeric antigen receptor $\mathrm{T}$ cells through constitutive CD40L expression. Mol Ther. (2015) 23:769-78. doi: $10.1038 / \mathrm{mt} .2015 .4$

30. Narayanan P, Lapteva N, Seethammagari M, Levitt JM, Slawin KM, Spencer DM. A composite MyD88/CD40 switch synergistically activates mouse and human dendritic cells for enhanced antitumor efficacy. J Clin Invest. (2011) 121:1524-34. doi: 10.1172/JCI44327

31. Foster AE, Mahendravada A, Shinners NP, Chang W-C, Crisostomo J, Lu A, et al. Regulated expansion and survival of chimeric antigen receptor-modified T cells using small molecule-dependent inducible MyD88/CD40. Mol Ther. (2017) 25:2176-88. doi: 10.1016/j.ymthe.2017.06.014

32. Mata M, Gerken C, Nguyen P, Krenciute G, Spencer DM, Gottschalk S. Inducible activation of MyD88 and CD40 in CAR T cells results in controllable and potent antitumor activity in preclinical solid tumor models. Cancer Discov. (2017) 7:1306-19. doi: 10.1158/2159-8290.CD-17-0263

33. Ahmed N, Brawley VS, Hegde M, Robertson C, Ghazi A, Gerken C, et al. Human epidermal growth factor receptor 2 (HER2) -specific chimeric antigen receptor-modified $\mathrm{T}$ cells for the immunotherapy of HER2positive sarcoma. J Clin Oncol. (2015) 33:1688-96. doi: 10.1200/JCO.2014.5 8.0225

34. Hurton LV, Singh H, Najjar AM, Switzer KC, Mi T, Maiti S, et al. Tethered IL-15 augments antitumor activity and promotes a stem-cell memory subset in tumor-specific T cells. Proc Natl Acad Sci USA. (2016) 113:E7788-97. doi: $10.1073 /$ pnas. 1610544113

35. Krenciute G, Prinzing BL, Yi Z, Wu MF, Liu H, Dotti G, et al. Transgenic expression of IL15 improves antiglioma activity of IL13Ralpha2-CAR T cells but results in antigen loss variants. Cancer Immunol Res. (2017) 5:571-81. doi: 10.1158/2326-6066.CIR-16-0376

36. Hegde M, Mukherjee M, Grada Z, Pignata A, Landi D, Navai SA, et al. Tandem CAR T cells targeting HER2 and IL13Ralpha2 mitigate tumor antigen escape. J Clin Invest. (2016) 126:3036-52. doi: 10.1172/JCI83416 
37. Bielamowicz K, Fousek K, Byrd TT, Samaha H, Mukherjee M, Aware N, et al. Trivalent CAR T-cells overcome interpatient antigenic variability in glioblastoma. Neuro Oncol. (2017) 20:506-18. doi: 10.1093/neuonc/nox182

38. Chmielewski M, Kopecky C, Hombach AA, Abken H. IL-12 release by engineered $\mathrm{T}$ cells expressing chimeric antigen receptors can effectively Muster an antigen-independent macrophage response on tumor cells that have shut down tumor antigen expression. Cancer Res. (2011) 71:5697-706. doi: 10.1158/0008-5472.CAN-11-0103

39. Pegram HJ, Lee JC, Hayman EG, Imperato GH, Tedder TF, Sadelain M, et al. Tumor-targeted $\mathrm{T}$ cells modified to secrete IL-12 eradicate systemic tumors without need for prior conditioning. Blood (2012) 119:4133-41. doi: 10.1182/blood-2011-12-400044

40. Koneru M, Purdon TJ, Spriggs D, Koneru S, Brentjens RJ. IL-12 secreting tumor-targeted chimeric antigen receptor $\mathrm{T}$ cells eradicate ovarian tumors in vivo. Oncoimmunology (2015) 4:e994446. doi: 10.4161/2162402X.2014.99 4446

41. Chmielewski M, Abken H. CAR T cells releasing IL-18 convert to T-Bet(high) FoxO1(low) effectors that exhibit augmented activity against advanced solid tumors. Cell Rep. (2017) 21:3205-19. doi: 10.1016/j.celrep.2017.11.063

42. Hu B, Ren J, Luo Y, Keith B, Young RM, Scholler J, et al. Augmentation of antitumor immunity by human and mouse CAR T cells secreting IL-18. Cell Rep. (2017) 20:3025-33. doi: 10.1016/j.celrep.2017.09.002

43. Kunert A, Chmielewski M, Wijers R, Berrevoets C, Abken H, Debets R. Intra-tumoral production of IL18, but not IL12, by TCR-engineered T cells is non-toxic and counteracts immune evasion of solid tumors. Oncoimmunology (2017) 7:e1378842. doi: 10.1080/2162402X.2017.1378842

44. Shum T, Omer B, Tashiro H, Kruse RL, Wagner DL, Parikh K, et al. Constitutive signaling from an engineered IL7 receptor promotes durable tumor elimination by tumor-redirected T cells. Cancer Discov. (2017) 7:123847. doi: 10.1158/2159-8290.CD-17-0538

45. Hsu C, Hughes MS, Zheng Z, Bray RB, Rosenberg SA, Morgan RA. Primary human T lymphocytes engineered with a codon-optimized IL-15 gene resist cytokine withdrawal-induced apoptosis and persist long-term in the absence of exogenous cytokine. J Immunol. (2005) 175:7226-34. doi: $10.4049 /$ jimmunol.175.11.7226

46. Leonard JP, Sherman ML, Fisher GL, Buchanan LJ, Larsen G, Atkins MB, et al. Effects of single-dose interleukin-12 exposure on interleukin-12-associated toxicity and interferon-gamma production. Blood (1997) 90:2541-8.

47. Di Stasi A, Tey SK, Dotti G, Fujita Y, Kennedy-Nasser A, Martinez C, et al. Inducible apoptosis as a safety switch for adoptive cell therapy. $N$ Engl J Med. (2011) 365:1673-83. doi: 10.1056/NEJMoa1106152

48. Zhang L, Feldman SA, Zheng Z, Chinnasamy N, Xu H, Nahvi AV, et al. Evaluation of gamma-retroviral vectors that mediate the inducible expression of IL-12 for clinical application. J Immunother. (2012) 35:430-9. doi: 10.1097/CJI.0b013e31825898e8

49. Zhang L, Morgan RA, Beane JD, Zheng Z, Dudley ME, Kassim SH, et al. Tumor-infiltrating lymphocytes genetically engineered with an inducible gene encoding interleukin-12 for the immunotherapy of metastatic melanoma. Clin Cancer Res. (2015) 21:2278-88. doi: 10.1158/1078-0432.CCR-14-2085

50. Wilkie S, Van Schalkwyk MC, Hobbs S, Davies DM, Van Der Stegen SJ, Pereira AC, et al. Dual targeting of ErbB2 and MUC1 in breast cancer using chimeric antigen receptors engineered to provide complementary signaling. $J$ Clin Immunol. (2012) 32:1059-70. doi: 10.1007/s10875-012-9689-9

51. Kloss CC, Condomines M, Cartellieri M, Bachmann M, Sadelain M. Combinatorial antigen recognition with balanced signaling promotes selective tumor eradication by engineered T cells. Nat Biotechnol. (2013) 31:71-5. doi: $10.1038 /$ nbt.2459

52. Roybal KT, Rupp LJ, Morsut L, Walker WJ, Mcnally KA, Park JS, et al. Precision tumor recognition by $\mathrm{T}$ cells with combinatorial antigen-sensing circuits. Cell (2016) 164:770-9. doi: 10.1016/j.cell.2016.01.011

53. Kagoya Y, Tanaka S, Guo T, Anczurowski M, Wang CH, Saso K, et al. A novel chimeric antigen receptor containing a JAK-STAT signaling domain mediates superior antitumor effects. Nat Med. (2018) 24:352-9. doi: 10.1038/nm.4478

54. Leen AM, Sukumaran S, Watanabe N, Mohammed S, Keirnan J, Yanagisawa R, et al. Reversal of tumor immune inhibition using a chimeric cytokine receptor. Mol Ther. (2014) 22:1211-20. doi: 10.1038/mt.2014.47

55. Liu X, Ranganathan R, Jiang S, Fang C, Sun J, Kim S, et al. A chimeric switch-receptor targeting PD1 augments the efficacy of second-generation
CAR T cells in advanced solid tumors. Cancer Res. (2016) 76:1578-90. doi: 10.1158/0008-5472.CAN-15-2524

56. Mohammed S, Sukumaran S, Bajgain P, Watanabe N, Heslop HE, Rooney $\mathrm{CM}$, et al. Improving chimeric antigen receptor-modified $\mathrm{T}$ cell function by reversing the immunosuppressive tumor microenvironment of pancreatic cancer. Mol Ther. (2017) 25:249-58. doi: 10.1016/j.ymthe.201 6.10 .016

57. Bajgain P, Tawinwung S, D'elia L, Sukumaran S, Watanabe N, Hoyos $\mathrm{V}$, et al. CAR $\mathrm{T}$ cell therapy for breast cancer: harnessing the tumor milieu to drive $\mathrm{T}$ cell activation. J Immunother Cancer (2018) 6:34. doi: 10.1186/s40425-018-0347-5

58. Wieser R, Attisano L, Wrana JL, Massague J. Signaling activity of transforming growth factor beta type II receptors lacking specific domains in the cytoplasmic region. Mol Cell Biol. (1993) 13:7239-47. doi: 10.1128/MCB.13.12.7239

59. Foster AE, Dotti G, Lu A, Khalil M, Brenner MK, Heslop HE, et al. Antitumor activity of EBV-specific $\mathrm{T}$ lymphocytes transduced with a dominant negative TGF-beta receptor. J Immunother. (2008) 31:500-5. doi: 10.1097/CJI.0b013e318177092b

60. Bollard CM, Tripic T, Cruz CR, Dotti G, Gottschalk S, Torrano V, et al. Tumorspecific T-cells engineered to overcome tumor immune evasion induce clinical responses in patients with relapsed hodgkin lymphoma. J Clin Oncol. (2018) 36:1128-39. doi: 10.1200/JCO.2017.74.3179

61. Sukumaran S, Watanabe N, Bajgain P, Raja K, Mohammed S, Fisher WE, et al. Enhancing the potency and specificity of engineered $\mathrm{T}$ cells for cancer treatment. Cancer Discov. (2018) 8:972-87. doi: 10.1158/2159-8290.CD-17-1298

62. Wilkie S, Burbridge SE, Chiapero-Stanke L, Pereira AC, Cleary S, Van Der Stegen SJ, et al. Selective expansion of chimeric antigen receptor-targeted Tcells with potent effector function using interleukin-4. J Biol Chem. (2010) 285:25538-44. doi: 10.1074/jbc.M110.127951

63. Davies DM, Foster J, Van Der Stegen SJ, Parente-Pereira AC, ChiaperoStanke L, Delinassios GJ, et al. Flexible targeting of ErbB dimers that drive tumorigenesis by using genetically engineered T cells. Mol Med. (2012) 18:565-76. doi: 10.2119/molmed.2011.00493

64. Schalkwyk MCIV, Papa SE, Jeannon J-P, Urbano TG, Spicer JF, Maher J. Design of a phase I clinical trial to evaluate intratumoral delivery of ErbB-targeted chimeric antigen receptor T-cells in locally advanced or recurrent head and neck cancer. Hum Gene Ther Clin Dev. (2013) 24:134-42. doi: 10.1089/humc.2013.144

65. Tang L, Zheng Y, Melo MB, Mabardi L, Castano AP, Xie YQ, et al. Enhancing T cell therapy through TCR-signaling-responsive nanoparticle drug delivery. Nat Biotechnol. (2018) 36:707-16. doi: 10.1038/nbt.4181

66. Eyquem J, Mansilla-Soto J, Giavridis T, Van Der Stegen SJ, Hamieh $\mathrm{M}$, Cunanan $\mathrm{KM}$, et al. Targeting a CAR to the TRAC locus with CRISPR/Cas9 enhances tumour rejection. Nature (2017) 543:113-7. doi: 10.1038/nature21405

67. Roth TL, Puig-Saus C, Yu R, Shifrut E, Carnevale J, Li PJ, et al. Reprogramming human $\mathrm{T}$ cell function and specificity with non-viral genome targeting. Nature (2018) 559:405-9. doi: 10.1038/s41586-018-0326-5

68. Mirzaei HR, Pourghadamyari H, Rahmati M, Mohammadi A, Nahand JS, Rezaei A, et al. Gene-knocked out chimeric antigen receptor (CAR) T cells: tuning up for the next generation cancer immunotherapy. Cancer Lett. (2018) 423:95-104. doi: 10.1016/j.canlet.2018.03.010

Conflict of Interest Statement: SG has patent applications in the field of cell and gene therapy for cancer, and receives research support from TESSA Therapeutics.

The remaining author declares that the research was conducted in the absence of any commercial or financial relationships that could be construed as a potential conflict of interest.

Copyright (C) 2019 DeRenzo and Gottschalk. This is an open-access article distributed under the terms of the Creative Commons Attribution License (CC BY). The use, distribution or reproduction in other forums is permitted, provided the original author(s) and the copyright owner(s) are credited and that the original publication in this journal is cited, in accordance with accepted academic practice. No use, distribution or reproduction is permitted which does not comply with these terms. 